\title{
Investigação do equilíbrio corporal em idosos
}

\author{
Investigation of the corporal balance in elderly people
}

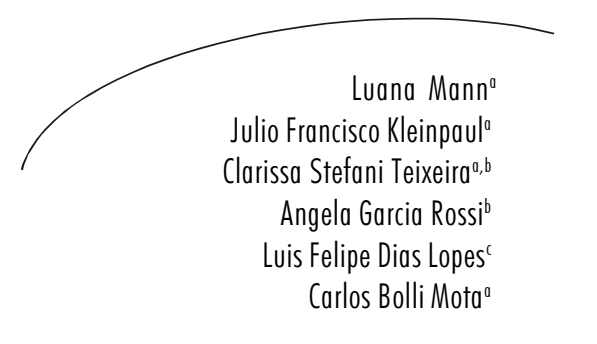

\section{Resumo}

Com o envelhecimento, o corpo humano passa por um peńodo de transformações que geram declínio de algumas capacidades físicas, tais como 0 equilíbrio. Este estudo objetivou comparar o equilíbrio corporal de idosos praticantes de hidroginástica e indivíduos adultos sedentários em diferentes bases de apoio, com a manipulação da visão. Foram avaliados 20 idosos praticantes de hidroginástica e 15 adultos sedentários. O equilíbrio foi coletado por meio de uma plataforma de força AMTI. As bases de apoio estudadas foram pés juntos, pés na largura do quadril e pé direito com afastamento anterior. 0 tempo de aquisição dos dados para cada tentativa foi de 10 segundos, a uma freqüência de $100 \mathrm{~Hz}$. Para comparação dos dados, foi utilizado teste $\mathrm{t}$ com nível de significância de 5\%. Comparando-se os grupos, não houve diferença estatisticamente significativa no equilíbrio nas três posições de bases de apoio, com e sem a utilização da informação visual, com exceção da variável amplitude do deslocamento do centro de pressão na direção médio-lateral, com os pés juntos e com os olhos abertos. Quando a informação visual foi manipulada, indivíduos de ambos os grupos apresentaram diferença estatisticamente significativa para a maioria das variáveis, destacando assim a importância da informação visual para idosos, mesmo estando-se fisicamente ativo. Inferiu-se, com os resultados obtidos, que a hidroginástica é um exercício recomendado para os idosos, uma vez que

Universidade Federal de Santa Maria

Santa Maria, RS, Brasil

a Centro de Educação Física - Laboratónio de Biomecânica

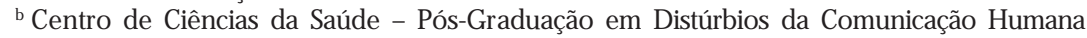

${ }^{c}$ Centro de Ciências Naturais e Exatas - Departamento de Estatística

\author{
Palavas-chave: \\ envelhecimento; \\ corpo humano; \\ aptidão física; \\ equilíbrio \\ musculosquelético; \\ idoso; atividade \\ motora; visão; \\ estudo comparativo
}

Comespondência / Comespondenœ

Luana Mann

E-mail: luanamann@gmail.com 
estes não se mostraram diferentes aos indivíduos mais novos, em relação ao equilíbrio corporal. Mas a hidroginástica não se mostrou favorável para melhorias na utilização do sistema visual.

\section{Abstract}

As people grow old, the human body undergoes for a long period of changes that cause a decline in some physical abilities, such as balance. This study aimed to compare the corporal balance of adult people who practice hydrogymnastics and sedentary elderly people in different support basis, with visual manipulation. It assessed 20 elderly people who practice hydrogymnastics and 15 sedentary adults. The balance was collected through a force platform AMTI. The support basis studied were feet together, feet in the width of the hip and right foot with anterior spacing. The time of data collection was 10 seconds for each attempt, in a $100 \mathrm{~Hz}$ frequency . For data companison it used the $t$ test with $5 \%$ of significance level . When comparing the hydrogymnastics and sedentary groups, there was no statistically meaningful difference in the variables in the three positions of support basis, with and without visual information, except for the variable amplitude of displacement of the pressure center in the medium-lateral direction, with feet together and open eyes. When the visual information was manipulated, individuals from both groups presented statistically meaningful difference for most variables, thus, calling attention for the importance of visual information for elderly people, even if they are physically active. It is possible to infer, through these results, that hydrogymnastics is recommended for elderly people, since they were not different from the younger individuals in relation to the corporal balance. However, hydrogymnastics did not seem to favor improvements in the use of the visual system.
Key words: aging;

human body; physical fitness; musculoskeletal equilibrium; aged; motor activity; vision; comparative study

\section{INTRODUÇÃO}

Com o envelhecimento, o corpo humano passa por um peńodo de transformações e degradações funcionais, que geram a perda de algumas capacidades físicas, tais como o equilíbrio. Manter o equilíbrio corporal requer a interação de uma série de informações provenientes de três sistemas: o vestibular, o somatossensonial e o visual. O sistema vestibularé sensível às acelerações lineares e angulares, enquanto o sistema somatossensonial é composto por vários re- œptores que perœbem a posição e a velocidade de todos os segmentos corporais, seu contato com objetos externos, inclusive 0 chão, e a onientação da gravidade. ${ }^{1}$ As informações visuais relacionam-se com a forma, cor e movimento dos objetos e do próprio corpo. No sistema visual, pode-se dizerque o envelhecimento compromete não só a acuidade visual, mas também restringe o campo visual e diminui a perœpção de profundidade. Tais alterações, segundo alguns autores, contribuem para a instabilidade corporal. ${ }^{2,3}$ 
Além das alterações no sistema biológico que ocorrem com o envelhecimento, as diferentes bases de apoio adotadas pelos indivíduos podem influenciar a manutenção do equilíbrio corporal. ${ }^{4,5,6}$ Diante de tais fatores, observa-se diminuição na qualidade de vida e prejuízo na realização das atividades da vida diánia, além de contribuir com o aumento dos gastos públicos decorrentes de elevados índices de quedas e de fraturas que atingem essa população. Devido ao grande número de fatores que afetam o equilíbrio, a prática de exercícios físicos está sendo sugenida como forte indicador de melhora de qualidade de vida, diminuição do nisco de quedas e fraturas nos idosos. ${ }^{7}$

A hidroginástica é apontada por Powers e Howley ${ }^{8}$ como fator de melhora nas capacidades físicas, aumento da coordenação, da agilidade, da sinestesia, da percepção, do esquema corporal, da velocidade de ação e reação, melhora no equilíbrio e na direcionalidade. Esses benefícios irão intervir na melhora da qualidade de vida para o idoso, possuindo caráter de prevenção e independência pessoal na vida cotidiana. Etchepare etal ${ }^{9}$ e Alves etal ${ }^{10}$ destacam que a hidroginástica é um excelente exercício físico para essa população, já que nela a ação de equilibrar-se é de grande importância para sua prática, podendo ser realizada por pessoas na terceira idade em virtude de ser uma prática com baixo impacto osteo-articular, possibilitando aos praticantes inúmeros benefícios, já que os mesmos, em certos casos, podem apresentar-se limitados fisicamente. ${ }^{11}$ As propriedades físicas da água, como a massa, o peso, a densidade relativa, a flutu- ação, a pressão hidrostática, a tensão superficial, e a viscosidade, são utilizadas como auxiliares na movimentação das articulações, utilizando os músculos de forma mais equilibrada e simétrica, e aumentando a vanedade dos movimentos corporais. ${ }^{12,13}$

Logo, resta-nos investigar se a hidroginástica é um exercício diferencial para a manutenção do equilíbrio corporal de idosos em diferentes bases de apoio, com e sem a informação visual. Diante disso, o presente estudo objetivou comparar o equilíbrio corporal de idosos praticantes de hidroginástica e indivíduos adultos sedentánios em diferentes bases de apoio, com a manipulação da visão.

\section{METODOLOGIA}

Idosos praticantes de hidroginástica e adultos sedentánios da comunidade foram convidados a participar do estudo. As coletas foram realizadas em ambiente de laboratório.

Foram avaliados 35 sujeitos sendo cinco do gênero masculino e 30 do gênero feminino. A participação no estudo foi voluntánia, sendo adotados como um dos criténos de exclusão a presença de problemas musculoesqueléticos e/ ou síndromes vestibulares. Como criténio de inclusão para o grupo de idosos, foi adotada idade supenor a 60 anos e experência na modalidade de hidroginástica entre 1 e 5 anos, com freqüência semanal de duas vezes, e a não-realização de outro exercício físico regular. 
Para o grupo de indivíduos adultos sedentánios, foi adotada idade a partir de 40 anos e a não-participação de nenhum tipo de exercício físico, desenvolvido nos últimos seis meses. Por meio de um questionánio com perguntas abertas, relacionado ao número eexercícios físicos desenvolvidos, tempo de prática e freqüência semanal, foram identificados os indivíduos sedentánios e os praticantes de exercícios regulares, que no presente estudo relacionaram-se com a hidroginástica. Dos 47 indivíduos que compareceram ao local de coleta, 12 foram excluídos do estudo por não satisfazerem algum destes critérios. Dois grupos foram selecionados: grupo 1- idosos praticantes de hidroginástica (HIDRO), totalizando 20 indivíduos, com idade média de 65,52 $\pm 3,74$ anos, peso corporal de 752,57 $\pm 129,83 \mathrm{~N}$ e estatura corporal de $1,57 \pm 0,07 \mathrm{~m}$; grupo 2- adultos sedentánios (SEDENT), totalizando 15 indivíduos com idade média de 58,53 $\pm 12,51$ anos, peso corporal de 682,42 \pm $80,60 \mathrm{~N}$ e estatura corporal de 1,59 $\pm 0,07$ $\mathrm{m}$. As aulas de hidroginástica praticadas pelos idosos são vinculadas a um projeto de extensão (Idoso, Natação e Saúde) junto ao Núcleo Integrado de Estudos e Apoio à Terceira Idade da Universidade Federal de Santa Maria, com freqüência de duas aulas semanais e com duração de 50 minutos

Este estudo foi aprovado pelo Comitê de Ética da Universidade Federal de Santa Maria, sob o número de protocolo 23081.007410/2007-17, no qual foram cumpridos os princípios éticos contidos na Declaração de Helsinque, além do atendimento à legislação vigente.
O equilíbrio foi coletado através de uma plataforma de força OR6-5 AMTI(Advanced MechanicalTemobgies, Inc). O posicionamento dosindivíduos foi padronizado, estando com os dois pés sobre a plataforma, com corpo na posição anatômica de referência, com olhar fixo em um ponto determinado e marcado na parede na altura dos olhos, distante dois metros da plataforma de força.

O equilíbnio corporal foi analisado através da amplitude do deslocamento do centro de pressão nas direções ântero-postenor (COPap) e médio lateral (COPml), e da média do deslocamento do centro de pressão nas direções ântero-posterior (DMap) e médio-lateral (DMml).

A partir dos sinais mensurados pela plataforma de força, a posição do centro de pressão (COP) é dada por:

$$
\begin{aligned}
& \mathrm{COP}_{\mathrm{ap}}=(\mathrm{My}-\mathrm{h} \cdot \mathrm{Fx}) / \mathrm{Fz} \\
& \mathrm{COP}_{\mathrm{m}-\mathrm{l}}=(\mathrm{Mx}-\mathrm{h} \cdot \mathrm{Fy}) / \mathrm{Fz}
\end{aligned}
$$

Onde:

$\mathrm{COP}_{\text {ap }}=$ coordenada do centro de pressão na direção ântero-postenior;

$\mathrm{COP}_{\mathrm{ml}}=$ coordenada do centro de pressão na direção médio-lateral;

$\mathrm{Mx}=$ momento em torno do eixo anteroposterior;

$\mathrm{My}=$ momento em torno do eixo médiolateral;

$\mathrm{Fx}=$ componente antero-postenior da força de reação do solo;

Fy = componente médio-lateral da força de reação do solo; 
Fz = componente vertical da força de reação do solo;

$\mathrm{h}=$ distância da supenfície até o centro geométrico da plataforma de força.

Os sujeitos foram avaliados em três diferentes bases de apoio de pés adaptadas do teste de Romberg ${ }^{14}$ assim como ilustra a figura 1: posição 1, com os pés juntos (P1); posição 2, com os pés na largura do quadril (P2); e posição 3, com o pé direito com afastamento anterior (P3). Os indivíduos tiveram tempo para ambientação ao laboratóno e as plataformas de força, bem como as diferentes bases de apoio.

O posicionamento dos pés foi padronizado no momento da primeira tentativa para cada indivíduo (com fita adesiva mar- cada sobre a plataforma, de acordo com a determinação da base de apoio), sendo a dimensão da base de apoio deteminada pela avaliação visual do avaliador e repetida nas demais tentativas, estando à distância padronizada de acordo com as caracteństicas físicas de cada indivíduo. Cada uma dessas posições foi avaliada em duas condições: olhos abertos (OA) e olhos fechados (OF), sendo realizadas três tentativas para cada posição e condição, totalizando 18 tentativas para cada indivíduo. A ordem de coleta, tanto para grupos como para as diferentes bases de apoio e condições visuais, foi randomizada por sorteio. $\mathrm{O}$ tempo de aquisição dos dados para cada tentativa foi de 10 s, após a estabilização visual do centro de pressão, a uma freqüência de aquisição de $100 \mathrm{~Hz}$.

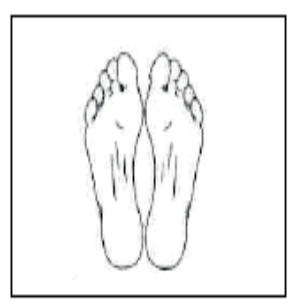

Posição1

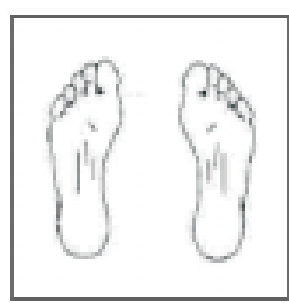

Posição 2

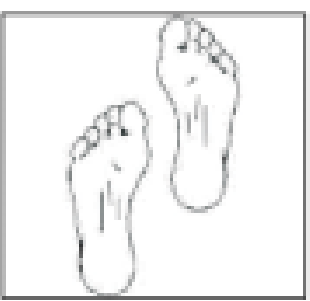

Posição 3

Figura 1 - Posicionamento dos pés durante a coleta de dados. Posição 1 (P1): pés juntos; posição 2 (P2): pés na largura do quadril e posição 3 (P3): pé direito com afastamento anterior. Adaptado de Teste de Romberg. ${ }^{14}$

Os dados foram submetidos à estatística descritiva. A normalidade dos dados foi venificada através do teste de Shapiro-Wilk, que mostrou que os dados podem ser considerados como tendo distribuição normal.
Primeiramente entre os grupos (HIDRO e SEDENT) nas distintas condições, com e sem informação visual, foram realizadas comparações entre as médias, em cada base de apoio, por meio do teste $t$ de Student. 
Em um segundo momento, também por meio do teste $t$ de Sudent, foram realizadas comparações intragrupos, ou seja, avaliou-se cada um dos grupos com e sem informação visual nas diferentes bases de apoio, a fim de identificar a influência da visão em cada um dos grupos. $\mathrm{O}$ valor de probabilidade de significância utilizado para todos os testes foi de 5\%.

\section{RESULTADOS}

Comparando-se os grupos HIDRO e SED ENT, apenas a variável COPml, na P1 e com os olhos abertos apresentou diferença estatisticamente significativa com probabilidade de significância de 0,02, conforme ilustra a tabela 1.

Tabela 1- Valores da média e do desvio-padrão das vanáveis COPap, COPml, DMap e DMml referentes a comparação dos grupos HIDRO e SEDENT nas três bases de apoio (P1, P2 e P3), com os olhos abertos $(\mathrm{AO})$ e com os olhos fechados (OF), e a probabilidade de significância (p) de cada vanável.

\begin{tabular}{|c|c|c|c|c|c|c|c|}
\hline \multirow{2}{*}{$\begin{array}{c}\text { Bases } \\
\text { de apoio }\end{array}$} & \multirow{2}{*}{ Condição } & \multirow{2}{*}{$\begin{array}{c}\text { Variáveis } \\
(\mathrm{cm})\end{array}$} & \multicolumn{2}{|c|}{ HIDRO } & \multicolumn{2}{|c|}{ SEDENT } & \multirow{2}{*}{$p^{*}$} \\
\hline & & & Média & DP & Média & DP & \\
\hline \multirow{8}{*}{ P1 } & \multirow{4}{*}{ OA } & COPap & 1,51 & 0,45 & 1,57 & 0,35 & 0,3573 \\
\hline & & COPml & 1,39 & 0,39 & 1,57 & 0,46 & 0,0211 \\
\hline & & DMap & 0,35 & 0,14 & 0,33 & 0,09 & 0,5401 \\
\hline & & DMml & 0,30 & 0,10 & 0,33 & 0,10 & 0,1429 \\
\hline & \multirow{4}{*}{ OF } & COPan & 202 & 072 & 213 & 81 & 3645 \\
\hline & & COPml & 1,82 & 0,51 & 1,90 & 0,45 & 0,3039 \\
\hline & & DMap & 0,40 & 0,15 & 0,42 & 0,17 & 0,3508 \\
\hline & & $\mathrm{DMml}$ & 0,34 & 0,12 & 0,37 & 0,11 & 0,2575 \\
\hline \multirow{8}{*}{ P2 } & \multirow{4}{*}{$\mathrm{OA}$} & COPap & 1,57 & 0,51 & 1,50 & 0,40 & 0.3407 \\
\hline & & COPml & 1,36 & 0,38 & 1,29 & 0,35 & 0.2235 \\
\hline & & DMap & 0,30 & 0,10 & 0,30 & 0,09 & 0.8954 \\
\hline & & $\mathrm{DMml}$ & 0,25 & 0,08 & 0,26 & 0,08 & 0.6906 \\
\hline & \multirow{4}{*}{ OF } & & & & & & \\
\hline & & COPap & $\begin{array}{l}1,86 \\
1,65\end{array}$ & 0,58 & $\begin{array}{l}1,78 \\
1,53\end{array}$ & $\begin{array}{l}0,72 \\
0,41\end{array}$ & $\begin{array}{l}0,4502 \\
0,1505\end{array}$ \\
\hline & & DMap & $\begin{array}{l}1,03 \\
0,37\end{array}$ & 0,13 & 0,35 & 0,14 & 0,3977 \\
\hline & & $\mathrm{DMml}$ & 0,30 & 0,10 & 0,28 & 0,08 & 0,1584 \\
\hline \multirow{9}{*}{ P3 } & \multirow{4}{*}{ OA } & COPap & 0,87 & 0,42 & 1,09 & 1,10 & 0,1074 \\
\hline & & COPml & 1,37 & 0,52 & 1,36 & 0,68 & 0,9010 \\
\hline & & DMap & 0,17 & 0,10 & 0,17 & 0,07 & 0,7907 \\
\hline & & $\mathrm{DMml}$ & 0,27 & 0,13 & 0,27 & 0,13 & 0,6854 \\
\hline & & & & & & & \\
\hline & \multirow{4}{*}{ OF } & COPap & 0,83 & 0,34 & 0,82 & 0,25 & 0,8488 \\
\hline & & COPml & 1,48 & 0,48 & 1,56 & 0,41 & 0,3336 \\
\hline & & DMap & 0,16 & 0,08 & 0,16 & 0,05 & 0,9317 \\
\hline & & $\mathrm{DMml}$ & 0,28 & 0,10 & 0,31 & 0,09 & 0,1000 \\
\hline
\end{tabular}

* $\mathrm{p}<0,05$ indicam diferença estatisticamente significativa, teste $\mathrm{t}$. 
De acordo com a tabela 2, que ilustra os valores da média e do desvio-padrão das vanáveis analisadas, comparando-se a influência da informação visual no equilíbrio em cada grupo separadamente submetido a diferentes bases de apoio, pode-se verificar que tanto indivíduos do grupo HIDRO quanto os do grupo SEDENT apresentaram diferença estatisticamente significativa para todas as variáveis analisadas na $\mathrm{P} 1 . \mathrm{Na}$ P2 as varáveis também foram estatisticamen- te significativas, com exceção da vaniável DMap no grupo HIDRO, e a variável DMml no grupo SEDENT.

Já na P3, quando o grupo HIDRO foi avaliado, as variáveis não apresentaram diferença estatisticamente significativa. No grupo SEDENT, durante a mesma posição, a única vanável que apresentou diferença estatisticamente significativa foi a DMml, com $\mathrm{p}=0,04$.

Tabela 2 - Valores da média e do desvio-padrão (DP) das variáveis COPap, COPml, DMap e DMml referentes a comparação da influência da informação visual em ambos os grupos, (HIDRO) e (SEDENT) nas três bases de apoio (P1, P2 e P3), e a probabilidade de significância (p) de cada variável.

\begin{tabular}{|c|c|c|c|c|c|c|c|}
\hline \multirow{2}{*}{$\begin{array}{c}\text { Bases } \\
\text { de apoio }\end{array}$} & \multirow{2}{*}{ Grupos } & \multirow{2}{*}{$\begin{array}{l}\text { Variáveis } \\
(\mathrm{cm})\end{array}$} & \multicolumn{2}{|c|}{$\mathrm{AO}$} & \multicolumn{2}{|c|}{ OF } & \multirow{2}{*}{$p^{*}$} \\
\hline & & & Média & DP & Média & $\mathrm{DP}$ & \\
\hline \multirow{9}{*}{ P1 } & \multirow{4}{*}{ HIDRO } & COPap & 1,51 & 0,45 & 2,02 & 0,72 & $<0,001$ \\
\hline & & COPml & 1,39 & 0,39 & 1,82 & 0,51 & $<0,001$ \\
\hline & & DMap & 0,35 & 0,14 & 0,40 & 0,15 & 0,03 \\
\hline & & $\mathrm{DMml}$ & 0,30 & 0,10 & 0,34 & 0,12 & 0,02 \\
\hline & & & & & & & \\
\hline & \multirow{4}{*}{ SEDENT } & COPap & 1,57 & 0,35 & 2,13 & 0,81 & $<0,001$ \\
\hline & & COPml & 1,57 & 0,46 & 1,90 & 0,45 & $<0,001$ \\
\hline & & DMap & 0,33 & 0,09 & 0,42 & 0,17 & $<0,001$ \\
\hline & & $\mathrm{DMml}$ & 0,33 & 0,10 & 0,37 & 0,11 & 0,04 \\
\hline \multirow{8}{*}{ P2 } & \multirow{4}{*}{ HIDRO } & COPap & 1,57 & 0,51 & 1,86 & 0,58 & $<0,001$ \\
\hline & & COPml & 1,36 & 0,38 & 1,65 & 0,51 & $<0,001$ \\
\hline & & DMap & 0,30 & 0,10 & 0,37 & 0,13 & 0,98 \\
\hline & & $\mathrm{DMml}$ & 0,25 & 0,08 & 0,30 & 0,10 & $<0,001$ \\
\hline & \multirow{4}{*}{ SEDENT } & COPap & 1,50 & 0,40 & 1,78 & 0,72 & 0,01 \\
\hline & & COPml & 1,29 & 0,35 & 1,53 & 0,41 & $<0,001$ \\
\hline & & DMap & 0,30 & 0,09 & 0,35 & 0,14 & 0,04 \\
\hline & & $\mathrm{DMml}$ & 0,26 & 0,08 & 0,28 & 0,08 & 0,15 \\
\hline \multirow{9}{*}{ P3 } & \multirow{4}{*}{ HIDRO } & COPap & 0,87 & 0,42 & 0,83 & 0,34 & 0,45 \\
\hline & & COPml & 1,37 & 0,52 & 1,48 & 0,48 & 0,16 \\
\hline & & DMap & 0,17 & 0,10 & 0,16 & 0,08 & 0,43 \\
\hline & & $\mathrm{DMml}$ & 0,27 & 0,13 & 0,28 & 0,10 & 0,71 \\
\hline & & & & & & & \\
\hline & \multirow{4}{*}{ SEDENT } & COPap & 1,09 & 1,10 & 0,82 & 0,25 & 0,06 \\
\hline & & COPml & 1,36 & 0,68 & 1,56 & 0,41 & 0,05 \\
\hline & & DMap & 0,17 & 0,07 & 0,16 & 0,05 & 0,14 \\
\hline & & $\mathrm{DMml}$ & 0,27 & 0,13 & 0,31 & 0,09 & 0,04 \\
\hline
\end{tabular}

* $\mathrm{p}<0,05$ indicam diferença estatisticamente significativa, teste $\mathrm{t}$ 


\section{DISCUSSÃO}

Estudos mostram que com a idade há uma diminuição no controle postural..$^{15,16,17}$ Essas considerações ficam evidenciadas no estudo de Manfio, Muniz e Rabello, ${ }^{18}$ que encontraram maiores valores de excursão do centro de pressão no grupo de idosos, quando comparados a indivíduos adultos com olhos abertos. 0 mesmo ocorreu no estudo de Silva etal, ${ }^{15}$ que verificou que jovens apresentaram maior estabilidade postural quando comparados aos idosos em relação à amplitude ântero-posterior e velocidade de deslocamento do centro de pressão, comprovando que este é maior com o avançar da idade.

No presente estudo, evidenciou-se que idosos que praticam hidroginástica mantêm valores similares a adultos sedentánios, demonstrando a importância da realização de um exercício físico regular na vida dos indivíduos. Na literatura científica, os exercícios físicos regulares são vistos como fundamentais na prevenção de quedas e no controle postural de idosos..$^{19}$ Alves etal., ${ }^{10}$ avaliando o efeito da hidroginástica em pré e pós-teste, encontraram melhoras no equilíbrio dinâmico de idosas e Etchepare etal. ${ }^{9}$ demonstraram a mesma tendência em idosasapós 20 sessões de exercícios.

Esta afirmação se confirma nas diferenças encontradas entre os dois grupos, na va nável $\mathrm{COPml}$, na $\mathrm{P} 1$, posição esta de menor base de apoio, na qual o grupo de idosos se mostrou com melhor estabilidade. Nas demais variáveis, a prática da hidroginástica não foi fator diferenciador em relação ao equilí- brio, mas deve-se salientar que indivíduos idosos que praticam exercícios físicos apresentam resultados similares de equilíbrio comparados a adultos sedentánios.

No presente estudo, pode-se destacar a influência da informação visual, já que a maionia das variáveis apresentou diferenças estatisticamente significativas em ambos os grupos, quando a presença ou supressão da informação visual foi comparada, indo ao encontro da literatura, pois diversos autoresapontam aumento da oscilação corporal quando é retirada alguma fonte de informação sensonial. ${ }^{16,20,21,17}$ Através da análise das vanáveis, perœbe-se que os valores encontrados são maiores quando a informação visual é manipulada.

No entanto, alguns autores sugerem que esta informação não é imprescindível ao equilíbrio..$^{22,23,24}$ No estudo de Ramos, ${ }^{7}$ no qual foi avaliado o equilíbrio de idosos em apoio bipodal após exercícios envolvendo capacidades neuromotoras, força, capacidade aeróbia, flexibilidade e exercícios respiratórios, a restrição da informação visual, a hidroginástica não proporcionou diferença significativa naárea de deslocamento do centro de pressão após a prática da atividade. Silva etal., ${ }^{15}$ em estudo com jovens adultose idosos, não encontraram diferenças quando a informação visual foi retirada.

A variável que apresentou os maiores valores de excursão do COP foi a COPap, tanto com os olhos abertos quanto com os olhos fechados, como já é previsto pela literatura. ${ }^{4,6,25,21}$ As maiores oscilações foram 
encontradas na direção ântero-posterior, tanto para a amplitude, como para o deslocamento médio do centro de pressão. ${ }^{26,20}$ Uma possível explicação estaria vinculada ao número de graus de liberdade na direção ântero-posterior, relacionado ao maior número de articulações envolvidas para manutenção do equilíbrio, quando comparada à direção médio-lateral, pois esta se resuminia basicamente a articulação do quadril. ${ }^{16}$

Porém durante a P3 observam-se, em ambos os grupos, valores superiores no sentido médio-lateral. Isto pode ser explicado pela diminuição da base de apoio, uma vez que ao deslocaro membro direito para frente, há um aumento na base no sentido ântero-posterior e uma diminuição no sentido médio-lateral.

Assim, pode-se dizer que a posição menos suscetível a interferência com a manipulação visual foi a P3, para ambos os grupos. No entanto, o DMml do grupo SEDENT mostrou-se com valores estatisticamente diferentes. Estes resultados podem inferir a interferência do exercício físico nesta posição para idosos que praticam hidroginástica, uma vez que, mesmo com diminuição da base de apoio no sentido médio-lateral, como no caso da P3, o grupo praticante de hidroginástica apresentou valores estatisticamente iguais com e sem a utilização da visão, o que não foi encontrado para o grupo de sedentánios.

Mesmo que Pernin etal. ${ }^{27}$ tenham encontrado como principal resultado o melhor controle postural em indivíduos esportis- tas, principalmente em relação à supressão visual, analisando-se especificamente os exercícios realizados durante a aula de hidroginástica para idosos, pode-se afirmar que não há ênfase nos estímulos visuais, estando as maiores alterações relacionadas ao sistema proprioceptivo, por meio do suporte fornecido pela água, que fornece maior independência da postura corporal. ${ }^{7}$

O presente estudo limitou-se a verificar se a prática regular de hidroginástica influencia no equilíbrio corporal de idosos em três diferentes bases de apoio e com a manipulação da informação visual. Não se investigou a influência de cada base de apoio sobre o equilíbrio do grupo de HIDRO e SEDENT.

Sugere-se a realização de estudos adicionais, a fim de se identificar a influência do tempo de prática, avaliando os idosos antes e após a modalidade, e em indivíduos adultos sedentánios com a mesma faixa etánia. A eficácia de diferentes modalidades, relacionando além do sistema visual, o sistema proprioceptivo e vestibular, também são avaliações pertinentes e importantes para realizar a prescrição do treinamento físico de idosos, tendo em vista as peculiaridades inerentes a esse público.

\section{CONCLUSÃO}

A partir dos resultados encontrados, pode-se sugenir que a hidroginástica, de forma geral, é uma boa atividade a ser praticada por idosos, uma vez que estes, quando 
comparados com indivíduos sedentánios de idades inferiores, mostraram-se com valores similares de equilíbrio corporal. A supressão ou manipulação do sistema sensonial causou maior dificuldade para a manutenção do equilíbrio corporal em ambos os grupos de idosos. Estes resultados indicam ser a informação visual uma importante fonte sensonial para a estabilidade postural em idosos.

Através dos resultados, percebe-se que 0 uso de diferentes bases de apoio pode seruma importante vanável de investigação em futuros estudos, em função de as mesmas estarem presentes no dia-a-dia de qualquer indivíduo.

\section{REFERÊNCIAS}

1. Winter D. Human balance and posture during standing and walking. Gait Posture 1995; 3(4):193 214.

2. Hobeika CP. Equilibrium and balance in the eldery. Ear Nose Throat Journal 1999; 78( 8): 558-66.

3. Bittar RSM, Pedalini MEB, Bottino MA, Formigoni LDG. Síndrome do Desequilíbrio do idoso. Pro Fono 2002; 14(1): 119-27.

4. Fialho CB, Vieira A, Loss J, Souza JL Amplitude de oscilação do centro de pressão em quatro bases de apoio durante a avaliação da postura ortostática - estudo preliminar. Anais do 9o Congresso Brasileiro de Biomecânica, 2001, Gramado, RS. Porto Alegre: Editora da UFRGS, 2001. p. 100-04.

5. Mochizuki L, Ávila AOV, Amadio AC. Interpretação preliminar de parâmetros biomecânicos na variação da posição dos apoios na manutenção da postura ereta. Anais do 8o Congresso Brasileiro de Biomecânica; 1999, Flonianópolis, SC. Florianópolis; 1999a. p.119 - 23.

6. Mochizuki L, Ávila AOV, Amadio AC. Resultados preliminares do estudo sobre a manutenção do equilíbrio em posturas unipodais. Anais do 8o Congresso
Brasileiro de Biomecânica; 1999, Florianópolis, SC. Florianópolis; 1999b. p. 251 - 54.

7. Ruoti RG, Morris DM, Cole AJ. Reabilitação aquática. São Paulo: Manole; 2000. 463p.

8. Powers SK, Howley ET. Fisiologia do exercício: teoria e aplicação ao condicionamento e ao desempenho. 5.ed. São Paulo: Manole; 2000. 527p.

9. Etchepare LS, Pereira EF, Graup S; Zinn JL. Terceira Idade: aptidão física de praticantes de hidroginástica. Revista Lecturas: EF e Deportes 2004; 1. [acesso 2006 out 10]. Disponível em: URL: <http:// www.efdeportes.com/ef65/ hidrog.htm $>$.

10. Alves RV, Mota J, Costa MC, Alves JGB. Aptidão física relacionada á saúde de idosos: influência da hidroginástica. Revista brasileira de medicina do esporte 2004; 10 (1): 31-7.

11. Carvalho Filho ET, Netto MP. Geniatria: fundamentos, clínica e terapêutica. 2. ed. São Paulo: Atheneu; 2006. 804 p.

12. Bonachela V. Manual básico de hidroginástica. 2. ed. Rio de Janeiro: Sprint; 1994. 94 p. 
13. Marques J, Pereira N. Hidroginástica: exercícios comentados, cinesiología aplicada à hidroginástica. Rio de Janeiro: Pereira; 1999.

14. Ramos BMB. Influência de um programa de atividade física no controle do equilíbrio de idosos. [monografia]. São Paulo: Universidade do Estado de São Paulo, Escola de Educação Física; 2003. 65 p.

15. Silva JB, Sousa PN, Lima ES, Teixeira LA. Comparação do controle postural entre Indivíduos adultos e idosos: dependência da tarefa e da informação visual. Anais do 5o Seminánio Intemacional sobre Atividades Físicas para a Terceira Idade Educação Física e Envelhecimento Perspectivas e Desafios, São Paulo, SP. São Paulo; 2002. p.175-78.

16. Freitas Júnior PB, Barela JA. Análise da postura ereta não perturbada de jovens adultos e idosos. Anais do $10^{\circ}$ Congresso Brasileiro de Biomecânica, 2003; Belo Honizonte, MG. Belo Honizonte: 2003. p. 36-9.

17. Teixeira CS, Lopez LFD, Rossi, AG, Mota $\mathrm{CB}$. The use of sight for the static balance maintenance in young people. The Fiep Bulletin 2007; 77: 636-39.

18. Manfio EF, Muniz MAS, Rabello BV. Relação entre equilíbrio estático e a força de reação do solo. Anais eletrônicos do $11^{\circ}$ Congresso Brasileiro de Biomecânica; 2005; João Pessoa, PB. João Pessoa; 2005.

19. Lord SR, Sherrington C, Menz HB. Falls in older people: risk factors and strategies for prevention. United Kingdom: Cambridge University; 2001.

20. Mann L, Teixeira CS, Lopes LFD, Mota CB. Avaliação do centro de força durante o equilíbrio estático em acadêmicos da educação física. Anais eletrônicos do $5^{0}$
Mostra de Iniciação Científica, Pósgraduação, Pesquisa e Extensão; 2005; Universidade de Caxias do Sul, Vacania, RS. Vacania; 2005.

21. Pranke GI, Mann L, Lemos LFC, Pasa SS. Equilíbrio corporal de idosos: suas relações com a visão. The Fiep Bulletin 2007; 77: 640-43.

22. Horak FB, Shupert CL. The role of the vestibular system in postural control. In: Herdman SJ, Whitney SL, Borello-France D F, editores. Vestibular rehabilitation 1994; 1(1): 22-46.

23. Shumway-Cook A, Woollacott M. Controle motor: teoria e aplicações práticas. 2.ed. Baruen, SP: Manole; 2003. 592p.

24. Wieczorek AS. Equilíbrio em adultos e idosos: relação entre tempo de movimento e acurácia durante movimentos voluntánios na postura em pé. [dissertação]. São Paulo: Universidade de São Paulo, Escola de Educação Física e Esporte; 2003. 83p

25. Oliveira EM, Estrázulas JA, Cruz A, Gomes R, Petry R, Guth VJ, Andrade MC, Melo SIL. MERCOMOVIMENTO.

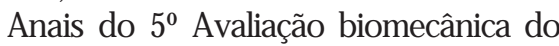
equilíbrio do idoso; 2004, Santa Maria, RS. Santa Maria; 2004. p. 69.

26. Prioli AC. Acoplamento entre informação visual discreta e contínua e oscilação corporal em idosos ativos e sedentários. [monografia]. Rio Claro, SP: Universidade Estadual Paulista "Júlio de Mesquita Filho"; 2003.75 p.

27. Perrin SD, Deviterne D, Perrot C, Constantinescu L Training improves the adaptation to changing visual conditions in maintaining human posture control in a test of sinusoidal oscillation of the support. Neurosci Lett 1998; 245( 3): 155-58. 
